\title{
Tesselations by Connection in Orders
}

\author{
Michel Couprie and Gilles Bertrand \\ Laboratoire A²SI, ESIEE Cité Descartes \\ B.P. 99, 93162 Noisy-Le-Grand Cedex France \\ \{coupriem, bertrand\}@esiee.fr
}

\begin{abstract}
The watershed transformation is a powerful tool for segmenting images, but its precise definition in discrete spaces raises difficult problems. We propose a new approach in the framework of orders. We introduce the tesselation by connection, which is a transformation that preserves the connectivity, and can be implemented by a parallel algorithm. We prove that this transformation possesses good geometrical properties. The extension of this transformation to weighted orders may be seen as a generalization of the watershed transformation.
\end{abstract}

Keywords: discrete topology, order, discrete distance, influence zones, watershed

\section{Introduction}

The watershed transformation [1] is a powerful tool for segmenting images. Initially introduced in the field of topography, the notion of watershed is often described in terms of steepest slope paths, watercourses and catchment basins. Here we prefer the following presentation, which is precise enough to be implemented by a computer program.

Consider a grayscale image as a topographic relief: the gray level of each point corresponds to its altitude. Watersheds may be obtained by piercing a hole at each minimum of this relief, and immersing the relief into a lake. The water will progressively fill up the different basins around each minimum. When the waters coming from different minima are going to merge, a dam is built to prevent the merging. At the end of this process, the set of points immersed by the water coming from one minimum $m_{i}$ is called the catchment basin associated to $m_{i}$, and the points that have not been immersed, and where dams have been built, constitute the watersheds.

This transformation may be seen as a set transformation, guided by the gray levels, which transforms the set of the points belonging to minima into the set of the points belonging to catchment basins. This set transformation has an important property: it preserves the connected components of the set (Fig. 1). On the other hand, the connected components of the complementary set are not preserved, as shown in Fig. $1\left(b_{2}, c_{2}\right)$. It is important to note that a topologypreserving transformation, such as an homotopic kernel [2] (which may be seen as an "ultimate skeleton"), should preserve both the connected components of 
the set and those of the complementary set (see Fig. $1\left(b_{1}, d_{1}\right.$ and $\left.b_{2}, d_{2}\right)$ ), and in $3 \mathrm{D}$, it should also preserve the tunnels of both the set and the complementary set.

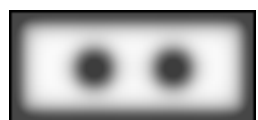

$\left(a_{1}\right)$

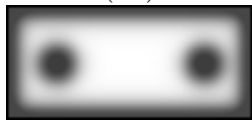

$\left(a_{2}\right)$

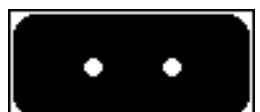

$\left(b_{1}\right)$

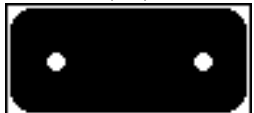

$\left(b_{2}\right)$

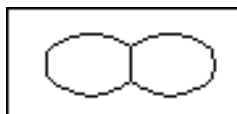

$\left(c_{1}\right)$

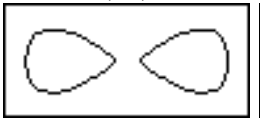

$\left(c_{2}\right)$

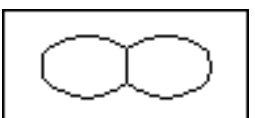

$\left(d_{1}\right)$

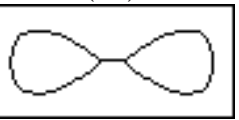

$\left(d_{2}\right)$

Fig. 1. $\left(a_{i}\right)$ : original images; $\left(b_{i}\right)$ : minima of $a_{i}$ (in white); $\left(c_{i}\right)$ : catchment basins of $a_{i}$ (in white); $d_{i}$ : an upper homotopic kernel of $b_{i}$ (in white)

Many definitions and algorithms for the watershed transformation have been proposed $[3,4,5,6,7]$. Particularly difficult is the problem of correctly specifying the watersheds that are located in plateaus, especially in the discrete spaces. Several authors have used a notion of distance in order to impose a "good centering" of the watersheds in plateaus, but in the usual discrete grids this centering is not always perfect. Their approach is based on the notion of influence zones: to a subset $X$ of $\mathcal{Z}^{n}$, which is composed of $k$ connected components $X_{1}, \ldots, X_{k}$, we can associate the influence zones $V_{1}, \ldots, V_{k}$ such that a point $x$ belongs to $V_{i}$ if $x$ is nearer from $X_{i}$ than from any other component $X_{j}$ of $X$. This constitutes a set transformation, which does not preserve any topological characteristic (as defined in the framework of digital topology [8]), not even the number of connected components: see Fig. 2.

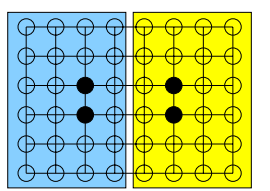

Fig. 2. Two regions (in black) and their respective influence zones (shaded) for the usual 4-distance. We see that the union of the two influence zones is 4connected, while the original set (black points) is not 4-connected

This paper extends a work presented in [9], in the framework of graphs. We propose a new approach based on the notion of order [10]. An order is equivalent to a discrete topological space (in the sense of Alexandroff [11]). In such a space, we prove that the influence zones transformation, defined thanks to the "natural" distance and applied to a closed set, preserves the connected components. We introduce the notion of uniconnected point, which allows to define a set transformation that preserves the connected components of the set: the tesselation by connection. From this set transformation, we derive a transformation 
on grayscale images, and more generally on weighted orders, that can be seen as a generalization of a watershed transformation. We propose some parallel algorithms to implement such transformations in orders and weighted orders. We also show that the link between these transformations and the influence zones transformation ensures that our algorithms produce a "well centered" result. In this paper, we give some examples based on orders that "modelize" the discrete grid $\mathcal{Z}^{n}$. Nevertheless, we emphasize the fact that all the presented properties and algorithms are valid for any order.

\section{Basic Notions}

In this section, we introduce some basic notions relative to orders (see also [10]).

If $X$ is a set, $\mathcal{P}(X)$ denotes the set composed of all subsets of $X$, if $S$ is a subset of $X, \bar{S}$ denotes the complement of $S$ in $X$. If $S$ is a subset of $T$, we write $S \subseteq T$, the notation $S \subset T$ means $S \subseteq T$ and $S \neq T$. If $\gamma$ is a map from $\mathcal{P}(X)$ to $\mathcal{P}(X)$, the dual of $\gamma$ is the map $* \gamma$ from $\mathcal{P}(X)$ to $\mathcal{P}(X)$ such that, for each $S \subseteq X, * \gamma(S)=\overline{\gamma(\bar{S})}$. Let $\delta$ be a binary relation on $X$, i.e., a subset of $X \times X$. We also denote by $\delta$ the map from $X$ to $\mathcal{P}(X)$ such that, for each $x$ of $X, \delta(x)=\{y \in X,(x, y) \in \delta\}$. We define $\delta^{\square}$ as the binary relation $\delta^{\square}=\delta \backslash\{(x, x) ; x \in X\}$.

An order is a pair $|X|=(X, \alpha)$ where $X$ is a set and $\alpha$ is a reflexive, antisymmetric, and transitive binary relation on $X$. An element of $X$ is also called a point. The set $\alpha(x)$ is called the $\alpha$-adherence of $x$, if $y \in \alpha(x)$ we say that $y$ is $\alpha$-adherent to $x$.

Let $(X, \alpha)$ be an order. We denote by $\alpha$ the map from $\mathcal{P}(X)$ to $\mathcal{P}(X)$ such that, for each subset $S$ of $X, \alpha(S)=\cup\{\alpha(x) ; x \in S\}, \alpha(S)$ is called the $\alpha$ closure of $S, * \alpha(S)$ is called the $\alpha$-interior of $S$. A subset $S$ of $X$ is $\alpha$-closed if $S=\alpha(S), S$ is $\alpha$-open if $S=* \alpha(S)$.

Let $(X, \alpha)$ be an order. We denote by $\beta$ the relation $\beta=\{(x, y) ;(y, x) \in \alpha\}$, $\beta$ is the inverse of the relation $\alpha$. We denote by $\theta$ the relation $\theta=\alpha \cup \beta$. The dual of the order $(X, \alpha)$ is the order $(X, \beta)$.

Note that $* \alpha(S)=\{x \in S ; \beta(x) \subseteq S\}$, and $* \beta(S)=\{x \in S ; \alpha(x) \subseteq S\}$.

The set $\mathcal{O}_{\alpha}$ composed of all $\alpha$-open subsets of $X$ satisfies the conditions for the family of open subsets of a topology, the same result holds for the set $\mathcal{O}_{\beta}$ composed of all $\beta$-open subsets of $X$; we denote respectively by $\mathcal{T}_{\alpha}=\left(X, \mathcal{O}_{\alpha}\right)$ and by $\mathcal{T}_{\beta}=\left(X, \mathcal{O}_{\beta}\right)$ these two topologies. These topologies are Alexandroff topologies, i.e., topologies such that every intersection of open sets is open [11].

An order $(X, \alpha)$ is countable if $X$ is countable, it is locally finite if, for each $x \in X, \theta(x)$ is a finite set. A $C F$-order is a countable locally finite order.

If $(X, \alpha)$ is an order and $S$ is a subset of $X$, the order relative to $S$ is the order $|S|=(S, \alpha \cap(S \times S))$.

Let $(X, \alpha)$ be a CF-order. Let $x_{0}$ and $x_{k}$ be two points of $X$. A path from $x_{0}$ to $x_{k}$ is a sequence $x_{0}, x_{1}, \ldots, x_{k}$ of elements of $X$ such that $x_{i} \in \theta\left(x_{i-1}\right)$, with $i=1, \ldots, k$. The number $k$ is called the length of the path. We consider the relation $\{(x, y)$; there is a path from $x$ to $y\}$. It is an equivalence relation, its 
equivalence classes are called the connected components of $X$. We say that $(X, \alpha)$ is connected if it has exactly one connected component.

Let $|X|=(X, \alpha)$ be a CF-order. The $\alpha$-rank of a point $x$ is the length of a longest $\alpha$-path having $x$ as origin. It is denoted by $r(x)$.

Let $(X, \alpha)$ be an order. An element $x$ such that $\alpha^{\square}(x)=\emptyset$ (i.e., such that $r(x)=0$ ) is said to be $\alpha$-terminal (for $X$ ).

\section{Orders Associated to $\mathcal{Z}^{n}$}

We give now a presentation of some orders which may be associated to $\mathcal{Z}^{n}[12,10]$.

Let $\mathcal{Z}$ be the set of integers. We consider the families of sets $H_{0}^{1}, H_{1}^{1}, H^{1}$ such that, $H_{0}^{1}=\{\{a\} ; a \in \mathcal{Z}\}, H_{1}^{1}=\{\{a, a+1\} ; a \in \mathcal{Z}\}, H^{1}=H_{0}^{1} \cup H_{1}^{1}$.

A subset $S$ of $\mathcal{Z}^{n}$ which is the Cartesian product of exactly $m$ elements of $H_{1}^{1}$ and $(n-m)$ elements of $H_{0}^{1}$ is called a $m$-cube of $\mathcal{Z}^{n}$. We denote $H^{n}$ the set composed of all $m$-cubes of $\mathcal{Z}^{n}, m=0, \ldots, n$.

An $m$-cube of $\mathcal{Z}^{n}$ is called a singleton if $m=0$, a unit interval if $m=1$, a unit square if $m=2$, a unit cube if $m=3$.

In this paper, the basic order associated to $\mathcal{Z}^{n}$ is the order $\left(H^{n}, \alpha\right)$, where $\alpha=\supseteq$, thus $y \in \alpha(x)$ if $x \supseteq y$. In Fig. 3(a), an example of a subset $S$ of $H^{2}$ is given. The object $S$ is made of two connected components $S_{1}$ (to the left) and $S_{2}$ (to the right). It may be seen that $S_{1}$ contains one singleton ( $\alpha$-terminal, $\alpha$-rank 0 ), two unit intervals ( $\alpha$-rank 1 ), and two unit squares ( $\beta$-terminals, $\alpha$-rank 2 ). In Fig. 3(b), an alternative representation of the same object is presented, we call it the array representation. We use the following conventions: a singleton is depicted by a circle $(\circ)$, a unit interval by a rectangle $(\square)$, and a unit square by a square $(\square)$.

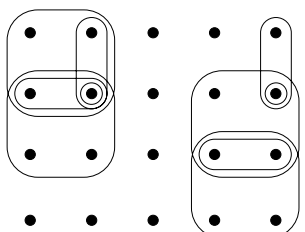

(a)

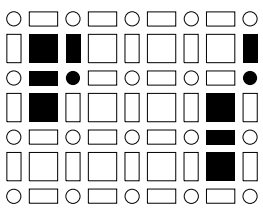

(b)

Fig. 3. (a): a subset $S$ of $H^{2}$, and (b): its array representation

In order to build consistent topological notions for a subset $S$ of $\mathcal{Z}^{n}$, we associate to $S$ a subset $\Psi(S)$ of $H^{n}$; thus we recover the structure of a (discrete) topological space by considering the order $\left(H^{n}, \supseteq\right)$. In this paper, the transformation $\Psi$ is chosen in such a way that the induced topological notions may be seen as "compatible" with the notions derived from the digital topology framework. A natural idea for defining $\Psi$ is to consider "hit or miss" transformations [13]. Thus we consider the set $S^{h}$ composed of all elements of $H^{n}$ which have a non-empty intersection with $S$. In a dual way, we consider the set $S^{m}$ composed of all elements of $H^{n}$ which are included in $S$ :

$\Psi^{h}(S)=S^{h}=\left\{x \in H^{n}, x \cap S \neq \emptyset\right\} ; \Psi^{m}(S)=S^{m}=\left\{x \in H^{n}, x \subseteq S\right\}$ 


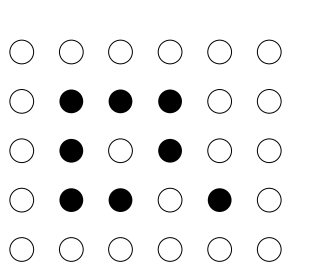

Fig. 4. (a): a subset $S$ of $\mathcal{Z}^{2}$ (black disks), (b): $S^{h}=\Psi^{h}(S), \stackrel{(c)}{(\text { c })}$ : $: S^{m}=\Psi^{m}(S)$

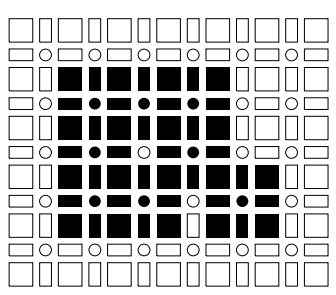

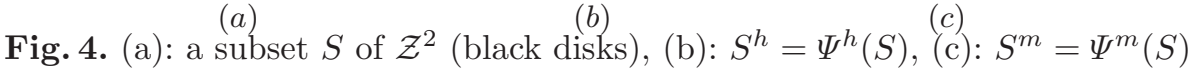

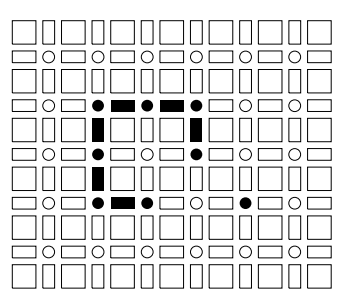

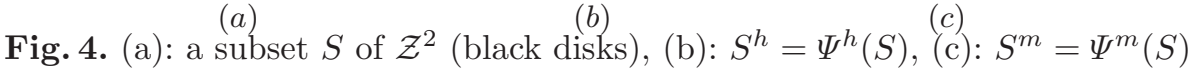

Fig. 4(a) presents an object $S$ of $\mathcal{Z}^{2}$ (in black). The adjacency used for the object is denoted by $a$, the adjacency used for the background is denoted by $\bar{a}$ (see [8]). Note that if $(a, \bar{a})=(4,8)$, then $S$ is composed of two connected components and $\bar{S}$ is connected. On the other hand if $(a, \bar{a})=(8,4)$, then $S$ is connected and $\bar{S}$ is composed of two connected components. The images $S^{h}$ and $S^{m}$ are shown in Fig. $4(\mathrm{~b})$ and (c) respectively. We note that in $S^{h}$ we retrieve the connectivity that corresponds to $(8,4)$, and in $S^{m}$ we retrieve the connectivity that corresponds to $(4,8)$. For a more complete discussion about a model of the digital topology notions in the framework of orders, see [14].

Note also that $S^{h}$ is always an $\alpha$-open set (i.e., a $\beta$-closed set), and that $S^{m}$ is always an $\alpha$-closed set.

\section{Distance and Influence Zones}

Let $(X, \alpha, \beta)$ be a CF-order, let $x$ and $y$ be two points of $X$. Let $\pi=\left(x_{0}, \ldots, x_{k}\right)$, with $x=x_{0}, y=x_{k}$, be a path from $x$ to $y$. The length of $\pi$ is denoted by $l(\pi)$ and is equal to $k$. We denote by $\Pi_{x, y}$ the set of all the paths from $x$ to $y$, and we define $d(x, y)=\min \left\{l(\pi), \pi \in \Pi_{x, y}\right\}$, the distance between $x$ and $y$. A path $\sigma$ from $x$ to $y$ such that $l(\sigma)=d(x, y)$ is a shortest path from $x$ to $y$.

It may be easily checked that $d$ is a (discrete) distance on $X$, that is, a map from $X \times X$ to $\mathcal{N}$ that verifies the properties of symmetry, separation $(d(x, y)=0$ implies $x=y)$ and triangular inequality $(d(x, y) \leq d(x, z)+d(z, y))$.

If $Y$ is a subset of $X$, we define $d(x, Y)=\min \{d(x, y), y \in Y\}$, and call this value the distance between $x$ and $Y$. A path $\sigma$ from $x$ to a point $y \in Y$ such that $l(\sigma)=d(x, Y)$ is called a shortest path from $x$ to $Y$.

Let $R$ be a subset of $X$, and let $\left\{R_{1}, \ldots, R_{m}\right\}$ be the connected components of $R$. For each $R_{i}, i=1, \ldots, m$, we define the influence zone $V_{i}$ associated to $R_{i}$ : $V_{i}=\left\{x \in X / \forall j \in[1, \ldots, m], j \neq i, d\left(x, R_{i}\right)<d\left(x, R_{j}\right)\right\}$.

Ideally, the influence zones should possess the following, desirable properties: first, they should be connected sets, and second, they should be mutually disconnected, i.e. any union of two different influence zones should be a non-connected set. If we consider the discrete plane $\mathcal{Z}^{2}$, and the classical 4- or 8-distance, the property of mutual disconnection is not verified (see Fig. 2).

Here, we prove that the influence zones associated to a family of $\alpha$-closed or $\beta$-closed subsets of a CF-order possess these two fundamental properties. We have to prove first these two intermediate lemmas: 
Lemma 1 Let $(X, \alpha, \beta)$ be a CF-order, and let $\pi=\left(x_{0}, \ldots, x_{k}\right)$ be a path. If $\pi$ is a shortest path from $x_{0}$ to $x_{k}$, then $\forall i=0, \ldots, k-2$ we have: $x_{i+1} \in \alpha^{\square}\left(x_{i}\right) \Rightarrow x_{i+2} \in \beta^{\square}\left(x_{i+1}\right)$, and $x_{i+1} \in \beta^{\square}\left(x_{i}\right) \Rightarrow x_{i+2} \in \alpha^{\square}\left(x_{i+1}\right)$.

Proof: clearly, $\forall i=0, \ldots, k-1 ; \quad x_{i} \neq x_{i+1}$. Suppose that $x_{i+1} \in \alpha^{\square}\left(x_{i}\right)$ and $x_{i+2} \notin \beta^{\square}\left(x_{i+1}\right)$. We know that $\pi$ is a path, hence we have $x_{i+2} \in \alpha^{\square}\left(x_{i+1}\right)$. Thus, $x_{i+2} \in \alpha^{\square}\left(x_{i}\right)$, and we see that the path $\left(x_{0}, \ldots, x_{i}, x_{i+2}, \ldots, x_{k}\right)$ is shorter than $\pi$, a contradiction.

Lemma 2 Let $(X, \alpha, \beta)$ be a $C F$-order, let $R$ be an $\alpha$-closed subset of $X$, let $x$ be a point of $X \backslash R$. Then, there exists a shortest path $\pi$ from $x$ to $R$ such that the last point $y$ of $\pi$ is an $\alpha$-terminal.

Proof: let $\pi$ be a shortest path from $x$ to $R$, let $w, y$ be the two last points of $\pi(y \in R)$. We have $y \in \alpha(w)$, because if we suppose $w \in \alpha(y)$, then $w$ belongs to $R$ ( $R$ is $\alpha$-closed), which contradicts the hypothesis " $\pi$ is a shortest path from $x$ to $R$ ".

Suppose that $y$ is not an $\alpha$-terminal, i.e. $\alpha(y) \neq \emptyset$. As $R$ is $\alpha$-closed, we have $\alpha(y) \subseteq R$ and $\alpha(y)$ contains $\alpha$-terminals, let $y^{\prime}$ be one of them. We have $y \in \alpha(w)$ and $y^{\prime} \in \alpha(y)$, thus $y^{\prime} \in \alpha(w)$. Let us consider the path $\pi^{\prime}$ identical to $\pi$ except that $y$ is replaced by $y^{\prime}$ : it is also a shortest path from $x$ to $R$, and its last point is an $\alpha$-terminal.

We are now ready to prove the aforementioned properties:

Property 3 Let $(X, \alpha, \beta)$ be a $C F$-order, let $R$ be a subset of $X$, let $R_{1}, \ldots, R_{m}$ be the connected components of $R$, and let $V_{1}, \ldots, V_{m}$ be the influence zones associated to $R_{1}, \ldots, R_{m}$, respectively. For each $i=1, \ldots, m$, the set $V_{i}$ is connected.

Proof: we shall prove that for any $x$ in $V_{i}$, a shortest path between $x$ and $R_{i}$ is entirely included in $V_{i}$. The property follows immediately from this result and from the connectedness of $R_{i}$.

Let $x \in V_{i}$. Following the definition of influence zones, there exists a shortest path $\pi$ from $x$ to $R_{i}$ such that $l(\pi)=d\left(x, R_{i}\right)$ and $l(\pi)<d\left(x, R_{j}\right), \forall j \in$ $[1, \ldots, m], j \neq i$. Let $y$ be any point in $\pi$, and suppose that $y \notin V_{i}$, which means that there is a $k \in[1, \ldots, m], k \neq i$, such that $d\left(y, R_{k}\right) \leq d\left(y, R_{i}\right)$.

As $\pi$ is a shortest path from $x$ to $R_{i}$, the subpath $\pi^{\prime}$ of $\pi$ from $y$ to $R_{i}$ is a shortest path from $y$ to $R_{i}$, hence $l\left(\pi^{\prime}\right)=d\left(y, R_{i}\right)$. Also, the subpath $\pi^{\prime \prime}$ of $\pi$ from $x$ to $y$ is a shortest path from $x$ to $y$, hence $l\left(\pi^{\prime \prime}\right)=d(x, y)$.

Let $\sigma$ be a shortest path from $y$ to $R_{k}$, we have $l(\sigma)=d\left(y, R_{k}\right)$ and hence $l(\sigma) \leq l\left(\pi^{\prime}\right)$. Then using the triangular inequality: $d\left(x, R_{k}\right) \leq l\left(\pi^{\prime \prime}\right)+l(\sigma) \leq$ $l\left(\pi^{\prime \prime}\right)+l\left(\pi^{\prime}\right)$, that is $d\left(x, R_{k}\right) \leq d\left(x, R_{i}\right)$, a contradiction.

Property 4 Let $(X, \alpha, \beta)$ be a CF-order, let $R$ be an $\alpha$-closed subset of $X$, let $R_{1}, \ldots, R_{m}$ be the connected components of $R$, and let $V_{1}, \ldots, V_{m}$ be the influence zones associated to $R_{1}, \ldots, R_{m}$, respectively. Then, the $V_{i}$ 's are mutually disconnected, i.e. $\forall i, j \in[1, \ldots, m], i \neq j, V_{i} \cup V_{j}$ is not connected. 
Proof: suppose that there is an $x$ in $V_{i}$ and a $y$ in $V_{j}$ such that $x \in \theta(y)$. We shall: a. prove that $d\left(x, R_{i}\right)=d\left(y, R_{j}\right)$, and b. raise a contradiction using lemmas 1 and 2 .

a. Suppose that $d\left(x, R_{i}\right)<d\left(y, R_{j}\right)$. Let $\pi$ be a shortest path from $R_{i}$ to $x$, and let $\sigma$ be a shortest path from $R_{j}$ to $y$, we have: $l(\pi)=d\left(x, R_{i}\right), l(\sigma)=$ $d\left(y, R_{j}\right)$. The sequence $\pi y$ is a path from $R_{i}$ to $y$, and we have $l(\pi y)=l(\pi)+1$, hence $l(\pi y) \leq l(\sigma)$. But $d\left(y, R_{i}\right) \leq l(\pi y)$ (by definition of $\left.d\right)$, hence $d\left(y, R_{i}\right) \leq$ $d\left(y, R_{j}\right)$, a contradiction with the fact that $y \in V_{j}$. A symmetric argument shows that $d\left(x, R_{i}\right)>d\left(y, R_{j}\right)$ is also false, hence $d\left(x, R_{i}\right)=d\left(y, R_{j}\right)$.

b. Let $\pi$ be a shortest path from $R_{i}$ to $x$, begining by an $\alpha$-terminal (see lemma 2) and ending by the sequence $\left(x^{\prime}, x\right)$. Let $\sigma$ be a shortest path from $R_{j}$ to $y$, begining by an $\alpha$-terminal and ending by the sequence $\left(y^{\prime}, y\right)$. These two paths having the same length (see a.), and beginning by $\alpha$-terminals, we deduce from lemma 1: $x \in \alpha\left(x^{\prime}\right) \Leftrightarrow y \in \alpha\left(y^{\prime}\right)$. Our hypothesis $x \in \theta(y)$ implies either $x \in \alpha(y)$ or $y \in \alpha(x)$. For example, if $x \in \alpha(y)$ and $y \in \alpha\left(y^{\prime}\right)$, then we have $x \in \alpha\left(y^{\prime}\right)$, hence $l(\sigma x)=l(\sigma)=l(\pi)$. This implies that $d\left(x, R_{j}\right) \leq d\left(x, R_{i}\right)$, a contradiction with the fact that $x \in V_{i}$. There are three other possibilities, that lead to a contradiction in similar ways.

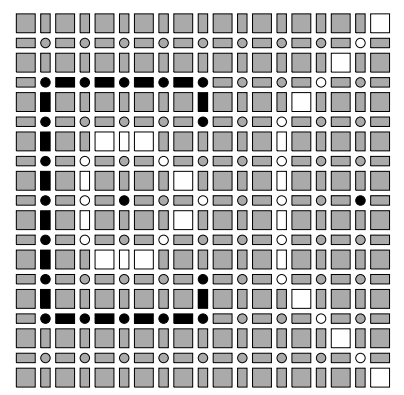

(a)

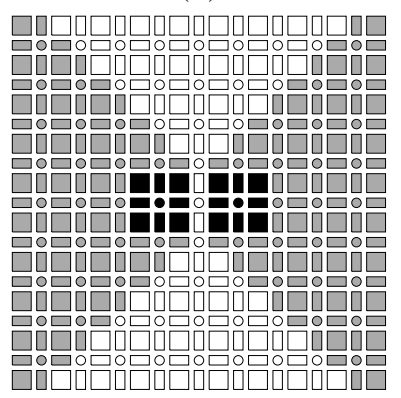

(c)

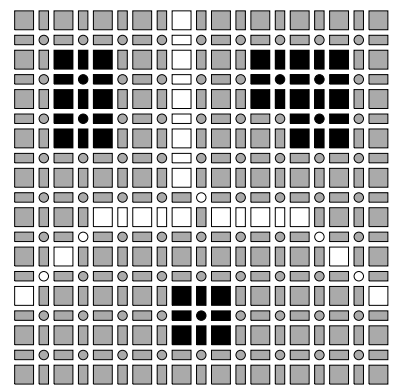

(b)

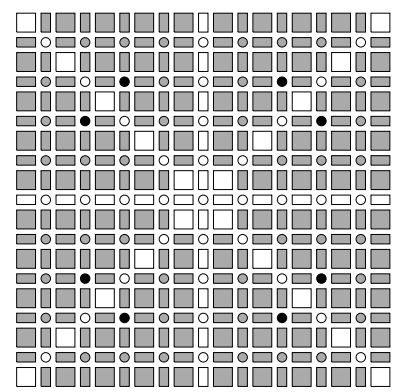

$(d)$

Fig. 5. Examples of families of $\alpha$-closed (a,d) or $\beta$-closed (b,c) sets (in black), and their influence zones (in gray) 


\section{Tesselation by Connection}

In Fig. 5, we show some examples of families of $\alpha$-closed (Fig. 5(a), in black) and $\beta$-closed (Fig. 5(b), in black) subsets of $H^{2}$, and their influence zones (in gray). In these cases, the complementary of the influence zones is "thin"; nevertheless, we cannot guarantee such a property in the general case. Fig. 5(c,d) shows counter-examples. In many image analysis applications, we need "thin" frontiers between the influence zones, this motivates the introduction of the following notions. Intuitively, a point is uniconnected if its addition preserves the connected components, in other terms, it preserves a connection in the sense of [15].

Let $(X, \alpha, \beta)$ be a finite $\mathrm{CF}$-order, let $R \subseteq X$, and let $x \in \bar{R}$. The point $x$ is uniconnected (for $R$ ) if the number of connected components of $R$ equals the number of connected components of $R \cup\{x\}$. The point $x$ is $\alpha$-uniconnected (for $R$ ) if $x$ is uniconnected for $R$ and if $\alpha(x) \cap R \neq \emptyset$.

We can easily see that a point $x \in \bar{R}$ is uniconnected if and only if $\theta(x)$ intersects exactly one connected component of $R$. A point $x \in \bar{R}$ is said to be multiconnected (for $R$ ) if $\theta(x)$ intersects at least two different connected components of $R$. It is isolated (for $R$ ) if $\theta(x) \cap R=\emptyset$.

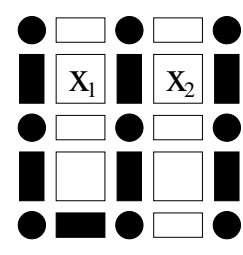

(a)

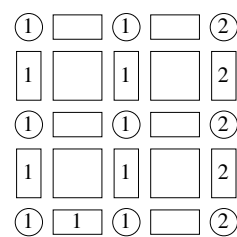

(b)

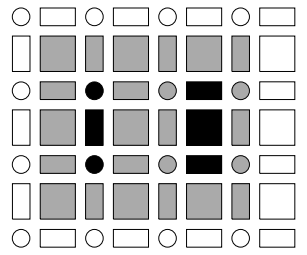

(c)

Fig. 6. (a): $x_{1}$ is uniconnected, $x_{2}$ is multiconnected; (b): labeling the components makes the local checking possible; (c): uniconnected points (in gray) cannot be added in parallel without changing the number of connected components

In Fig. 6, we can see that the information contained in $\theta(x)$ is not sufficient to check whether a point $x$ is uniconnected or multiconnected. On the other hand, if we assume that the points of each connected component of $R$ are labeled with an index which represents this component, then we can check whether a point $x$ is uniconnected or not by counting the number of different indexes carried by the points in $\theta(x)$ (see Fig. 6(b)). Furthermore, a uniconnected point $x$ is $\alpha$ uniconnected if $\alpha(x)$ contains at least one labeled point.

Let $R \subseteq X$, we say that $T \subseteq X$ is a thickening by connection of $R$ if $T$ may be derived from $R$ by iterative addition of uniconnected points. We say that $T$ is a tesselation by connection of $R$ if $T$ is a thickening by connection of $R$ and if all the points of $X \backslash T$ are not uniconnected.

In general, there are several tesselations by connection for a set $R$. This is due to the iterative nature of the definition: depending on the order of selection of the uniconnected points, one can get different results. Nevertheless, in many applications we want to obtain a "well centered" result, that could be uniquely 
defined. This is why we introduce, by the way of a parallel algorithm, a particular tesselation by connection that possesses good geometrical properties.

We can see in Fig. 6(c) that, in general, uniconnected points cannot be added in parallel to a set $R$ without changing the number of connected components of $R$. In fact, $\alpha$-uniconnected points can indeed be added in parallel to $R$ without changing the number of connected components of $R$. This can be proved by induction thanks to the following property.

Property 5 Let $(X, \alpha, \beta)$ be a CF-order, let $R \subseteq X$, and let $x, y \in \bar{R}$ be two $\alpha$-uniconnected points for $R$. Then, $x$ is $\alpha$-uniconnected for $R \cup\{y\}$.

Proof: if $y \notin \theta(x)$, then the property is obvious. If $y \in \alpha(x)$ or $x \in \alpha(y)$, then the component of $R$ which is $\alpha$-adherent to $y$ is clearly the same as the component of $R$ which is $\alpha$-adherent to $x$. Thus in both cases, adding the point $y$ to $R$ does not change the fact that $x$ is $\alpha$-uniconnected.

The following algorithm computes a tesselation by connection of a closed subset of $X$.

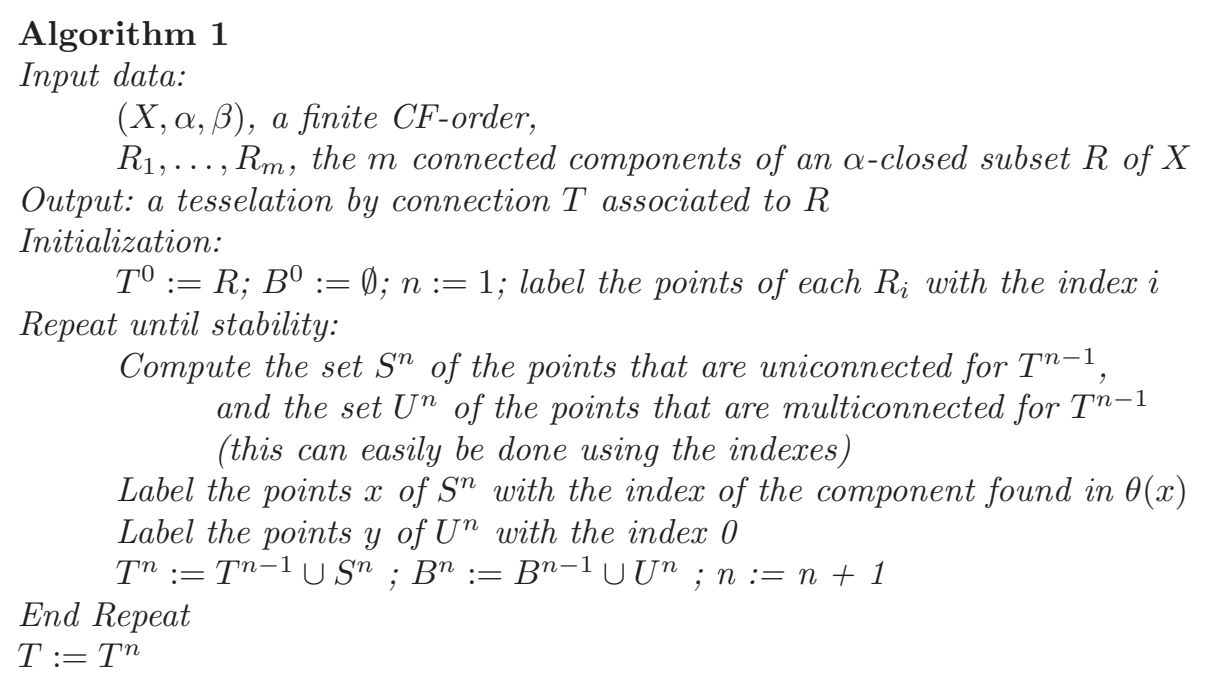

It can be easily seen that for any even (resp. odd) value of $n$, the set $T^{n}$ is $\alpha$-closed (resp. $\beta$-closed) in $\left|X \backslash B^{n}\right|$. From this, it follows that $S^{n+1}$ is only composed of $\alpha$-uniconnected (resp. $\beta$-uniconnected) points if $n$ is even (resp. odd). Thus (Prop. 5), the result $T$ of this algorithm is a thickening by connection of the original $\alpha$-closed set $R$. In addition, at the end of this algorithm no uniconnected point remains. Thus, the result $T$ is a tesselation by connection of the original set $R$.

The following property makes a link between the tesselation by connection computed by algorithm 1 an the influence zones, ensuring that this tesselation by connection is "well centered".

The following lemma and the following property derive from the fact that the labeling process is guided by a breadth-first strategy. 
Lemma 6 Every point $x$ that receives an index during the step $n$ of the algorithm, is such that $d(x, R)=n$.

Every point $x$ that receives an index $i>0$ during the step $n$ of the algorithm, is such that $d\left(x, R_{i}\right)=n$.

Property 7 Let $(X, \alpha, \beta)$ be a CF-order, let $R_{1}, \ldots, R_{m}$ be the $m$ connected components of an $\alpha$-closed subset $R$ of $X$, let $V_{1}, \ldots, V_{m}$ be the influence zones associated to $R_{1}, \ldots, R_{m}$, respectively, and let $T_{1}, \ldots, T_{m}$ be the connected components of the tesselation by connection of $R$ computed by algorithm 1 . Then we have : $\forall i=1, \ldots, m, V_{i} \subseteq T_{i}$.

This property establishes that the influence zones are included in the components of the tesselation by connection computed by algorithm 1 . The converse is true only in some cases, like in Fig. $5(\mathrm{a}, \mathrm{b}, \mathrm{d})$; but it is not true in general, as shown by the counter-examples of Fig. 7 .

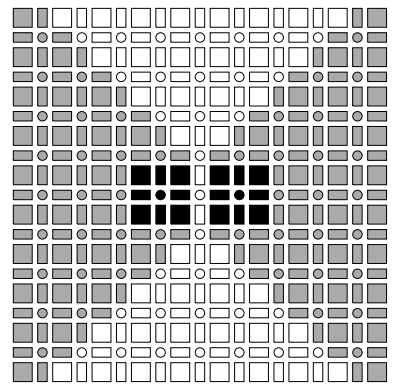

(a)

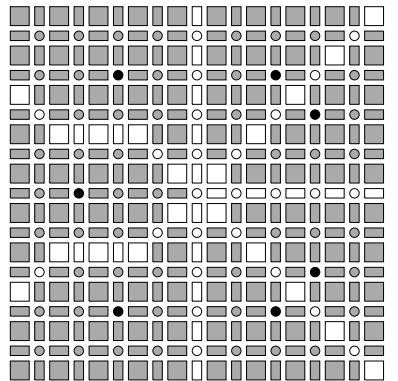

(b)

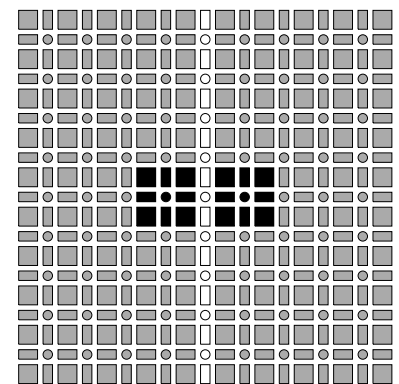

$\left(a^{\prime}\right)$

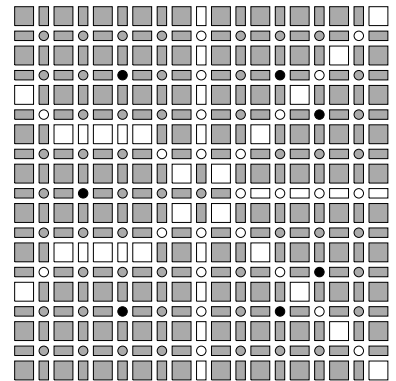

$\left(b^{\prime}\right)$

Fig. 7. (a,b): some objects (in black) and their influence zones (in gray). (a',b'): the same objects (in black) and their tesselation by connection (in gray)

\section{Tesselation by Connection for Weighted Orders}

In this section, we extend the notions of uniconnected point and tesselation by connection to weighted orders. A tesselation by connection for weighted orders may be considered as a generalization of the watershed transformation[1]: the result of the proposed transformation is a function, whereas the result of a 
watershed transformation is a set. We recover a set which corresponds to the complementary of the watersheds, by extracting the regional maxima of the tesselation by connection.

Let $|X|=(X, \alpha, \beta)$ be a finite $\mathrm{CF}$-order, and let $W$ be a mapping from $X$ to $\mathcal{Z}$. The couple $(|X|, W)$ is called a weighted order on $|X|$. For applications to digital image processing, $W(x)$ typically represents the graylevel of the point $x$. We denote by $W_{k}$, and call the cross-section of $W$ at level $k$, the set $W_{k}=\{x \in$ $X, W(x) \geq k\}$ with $k \in \mathcal{Z}$. The weighted order $(|X|, W)$ is $\alpha$-closed if, $\forall k \in \mathcal{Z}$, the order $\left|W_{k}\right|$ is $\alpha$-closed.

Let $(|X|, W)$ be a weighted order, and let $x \in X$. The point $x$ is uniconnected for $W$ if $x$ is uniconnected for $W_{k+1}$, with $k=W(x)$.

Let $(|X|, W)$ and $(|X|, M)$ be weighted orders, we say that $M$ is a thickening by connection of $W$ if $M$ may be derived from $W$ by iteratively selecting a uniconnected point $x$ and raising its value $W(x)$ by one. We say that $M$ is a tesselation by connection of $W$ if $M$ is a thickening by connection of $W$ and if all the points of $X$ are not uniconnected for $M$.

\section{Algorithm 2}

Input data: $(|X|, W)$, a finite $\alpha$-closed weighted order, Output: a tesselation by connection $M$ associated to $W$.

Initialization: Let $L=\left(k_{0}, \ldots, k_{s}\right)$ the list of values taken by $W(x)$,

For all $i$ from 1 to $s$ sorted in increasing order.

Compute the tesselation by connection $Z$ of $W_{k_{i}}$ in the order induced by $W_{k_{i-1}}$, using algorithm 1

End For

For all $z \in Z \backslash W_{k_{i-1}}$ do $W[z]:=k_{i}$; End For

$M:=W$

The following transformations (introduced in [16]) may be used to construct a closed weighted order from a grayscale image.

Let $\mathcal{F}$ be the set of functions from $\mathcal{Z}^{n}$ to $\mathcal{N}$. Let $\mathcal{W}$ be the set of functions from $\mathcal{H}^{n}$ to $\mathcal{N}$. We define the transformations $\Psi^{h}$ and $\Psi^{m}$ from $\mathcal{F}$ to $\mathcal{W}$ which associate to each $F$ in $\mathcal{F}$ the functions $W^{h}$ and $W^{m}$ respectively, defined by:

$\forall x \in \mathcal{H}^{n}, W^{m}(x)=\min \left\{F(y), y \in \mathcal{Z}^{n},\{y\} \in \alpha(x)\right\}$

$\forall x \in \mathcal{H}^{n}, W^{h}(x)=\max \left\{F(y), y \in \mathcal{Z}^{n},\{y\} \in \alpha(x)\right\}$

It can be easily seen that $\left(|X|, W^{m}\right)$ is an $\alpha$-closed weighted order, and that $\left(|X|, W^{h}\right)$ is a $\beta$-closed weighted order. In Fig. 8, we show the weighted order obtained by applying $\Psi^{m}$ to a "real" image, and the tesselation by connection of this weighted order computed by algorithm 2 .

\section{References}

1. S. Beucher, Ch. Lantuejoul, "Use of Watersheds in Contour Detection", Proc. Int. Workshop on Image Processing, Real-Time Edge and Motion Detection/Estimation, Rennes, France, 1979. 15, 24 


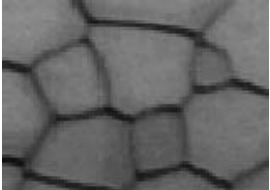

$(a)$

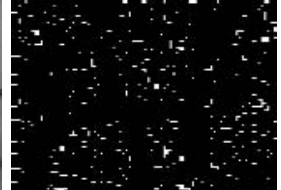

$\left(a^{\prime}\right)$

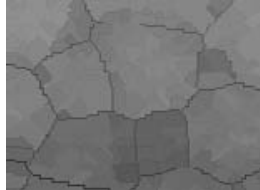

(b)

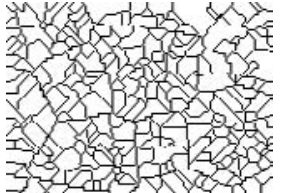

$\left(b^{\prime}\right)$

Fig. 8. (a): a weighted order obtained thanks to the transformation $\Psi^{m}$; (b): tesselation by connection of $(a) ;\left(a^{\prime}, b^{\prime}\right)$ : regional maxima of $(a, b)$ respectively

2. C. Lohou, G. Bertrand, "Poset approach to 3D parallel thinning", SPIE Vision Geometry VIII., Vol. 3811, pp. 45-56, 1999. 15

3. S. Beucher, "Segmentation d'images et morphologie mathématique", PhD Thesis, École des Mines, Paris, 1990. 16

4. L. Vincent, P. Soille, "Watersheds in Digital Spaces: An Efficient Algorithm Based on Immersion Simulations", IEEE Trans. on Pattern Analysis and Machine Intelligence, Vol. 13, No. 6, pp. 583-598, 1991. 16

5. F. Prêteux, "Watershed and Skeleton by Influence Zones: A Distance-Based Approach", Journal of Mathematical Imaging and Vision, No. 1, pp. 239-255, 1992. 16

6. S. Beucher and F. Meyer, "The morphological approach to segmentation: the watershed transformation", Mathematical Morphology in Image Processing, Chap. 12, pp. 433-481, Dougherty Ed., Marcel Dekker, 1993. 16

7. F. Meyer, "Topographic distance and watershed lines", Signal Processing, No. 38, pp. 113-125, 1994. 16

8. T. Y. Kong and A. Rosenfeld, "Digital topology: introduction and survey", Comp. Vision, Graphics and Image Proc., 48, pp. 357-393, 1989. 16, 19

9. M. Couprie and G. Bertrand: "Topological Grayscale Watershed Transformation" SPIE Vision Geometry V Proceedings, Vol. 3168, pp. 136-146, 1997. 16

10. G. Bertrand, "New notions for discrete topology", 8th Conf. on Discrete Geom. for Comp. Imag., Vol. 1568, Lect. Notes in Comp. Science, Springer Verlag, pp. 218228, 1999. 16, 17, 18

11. P. Alexandroff, "Diskrete Räume", Mat. Sbornik, 2, pp. 501-518, 1937. 16, 17

12. E. Khalimsky, R. Kopperman, P. R. Meyer, "Computer Graphics and Connected Topologies on Finite Ordered Sets", Topology and its Applications, 36, pp. 1-17, 1990. 18

13. J. Serra, Image Analysis and Mathematical Morphology, Academic Press, 1982. 18

14. G. Bertrand, M. Couprie, "A model for digital topology", 8th Conf. on Discrete Geom. for Comp. Imag., Vol. 1568, Lect. Notes in Comp. Science, Springer Verlag, pp. 229-241, 1999. 19

15. G. Matheron, J. Serra, Strong filters and connectivity, in Image Analysis and Mathematical Morphology, Vol. II: Theoretical Advances, Chap. 7, pp. 141-157, Serra J. ed., Academic Press, 1988. 22

16. V. A. Kovalevsky, "Finite Topology as Applied to Image Analysis", Computer Vision, Graphics, and Image Processing, 46, pp. 141-161, 1989. 25 